\title{
The converse of the mean value theorem may fail generically
}

\section{The Converse of the Mean Value Theorem May Fail Generically}

\section{J. M. Borwein and Xianfu Wang}

In [4] Tong and Braza observed the following converse to the classical mean value theorem.

Theorem 1. Let $f$ be a function continuous on $[a, b]$ and differentiable on $(a, b)$ and let $c$ be a given point in $(a, b)$. Then

(1) Weak Form: If $f^{\prime}(c)$ is not a total extremum value on $(a, b)$, i.e., $f^{\prime}(c) \neq$ $\sup \left\{f^{\prime}(x) \mid x \in(a, b)\right\}$ and $f^{\prime}(c) \neq \inf \left\{f^{\prime}(x) \mid x \in(a, b)\right\}$, then there is some subinterval $\left(a_{1}, b_{1}\right) \subset(a, b)$ such that $f^{\prime}(c)=\left(f\left(b_{1}\right)-f\left(a_{1}\right)\right) /\left(b_{1}-a_{1}\right)$.

(2) Strong Form: If $f^{\prime}(c)$ is not a local extremum value of $f^{\prime}(x)$ on $(a, b)$ and if $c$ is not an accumulation point of the set $A_{c}:=\left\{x \in(a, b) \mid f^{\prime}(x)=f^{\prime}(c)\right\}$, then there is a subinterval $\left(a_{1}, b_{1}\right) \subset(a, b)$ such that $a_{1}<c<b_{1}$ and $f^{\prime}(c)=$ $\left(f\left(b_{1}\right)-f\left(a_{1}\right)\right) /\left(b_{1}-a_{1}\right)$.

They gave an example of a differentiable function with a countably infinite number of points at which Theorem 1 fails. They ask: Could these points be dense? Is it possible to have an uncountable number of such points?

The purpose of this note is to show that the converse may fail generically. We use a classical and well-known example to illustrate this. We follow the construction in $\left[\mathbf{1}\right.$, p. 205] and [3, pp. 215-216]. The function $f(x):=(x-a)^{\frac{1}{3}}$ has an infinite derivative at $x=a$, and a finite derivative elsewhere. We use $Q$ to denote the set of rational numbers. Let $q_{1}, q_{2}, \ldots$ be an enumeration of $Q \cap[0,1]$, and for each $n \in N$ let $f_{n}(x):=\left(x-q_{n}\right)^{\frac{1}{3}}$. Let

$$
F(x):=\sum_{n=1}^{\infty} \frac{f_{n}(x)}{10^{n}} .
$$

Then $F$ is absolutely continuous on $[0,1]$. Since each $f_{n}$ is strictly increasing, so is $F$. It can be shown that

$$
F^{\prime}(x)=\sum_{n=1}^{\infty} \frac{f_{n}^{\prime}(x)}{10^{n}}=\sum_{n=1}^{\infty} \frac{\left(x-q_{n}\right)^{-\frac{2}{3}}}{3 \cdot 10^{n}}
$$

In particular $F^{\prime}(x)=\infty$ for all $x \in Q \cap[0,1]$.

Let $g(x):=F(x)+x$. Then $g^{\prime}(x)=F^{\prime}(x)+1$ on $[0,1]$ and $g$ is a homeomorphism from $[0,1]$ to a nondegenerate interval $[a, b]$. Define $h$ by $h(x):=g^{-1}(x)$, that is, the inverse function of $g$. Then $h$ is strictly increasing and continuous on $[a, b]$, and $h^{\prime}=0$ on a dense set of $[a, b]$. Since $g^{\prime}(x) \geq 1$ for all $x \in[0,1]$ $h^{\prime}(x) \leq 1$ for all $x \in[a, b]$. Hence we have constructed a strictly increasing function $h$ on $[a, b]$ such that $h$ is differentiable, $0 \leq h^{\prime}(x) \leq 1$ for all $x \in[a, b]$, and $\left\{x: h^{\prime}(x)=0\right\}$ is dense in $[a, b]$; thus $h$ is a globally Lipschitz function on $[a, b]$. Because $h^{\prime}$ is a Baire-1 function (the pointwise limit of a sequence of continuous functions), $h^{\prime}$ is continuous except at the points of a set of the first 
category $[1$, p. 22]. Hence, the set

$$
D:=\left\{x \in[a, b] \mid h^{\prime}(x)=0\right\}
$$

is a residual subset of $[a, b]$. In particular, it is dense and uncountable. Since $h$ is strictly increasing, $(h(x)-h(y)) /(x-y)>0$ for all $x, y \in[a, b]$ with $x \neq y$. Then, every tangent line has zero slope at $x \in D$ but every possible secant line has a positive slope, and so $h$ fails both the strong and weak forms of Theorem 1 on $D$.

In contrast, we observe that the Lebesgue measure of $D$ is zero. This follows from the observation that $h$ has zero derivative at $g(x)$ if and only if the derivative of $g$ at $x$ is infinite. The set of points at which $g$ has infinite derivative is at most of zero measure. Indeed, one can use Denjoy-Young-Saks's theorem [1, p. 322] to show that, for every finite function $f$, the set $\left\{x: f^{\prime}(x)=x\right\}$ is of Lebesgue measure 0 . Since $g$ is absolutely continuous, $g$ maps null sets to null sets.

Finally one can verify that $f:(-\infty, 1 / 2] \mapsto R$ defined by

$$
f(x):= \begin{cases}x^{3} \sin \frac{1}{x}+\frac{x^{2}}{2} & \text { if } 0<x \leq \frac{1}{2} \\ 0 & \text { if } x \leq 0,\end{cases}
$$

fails the strong form of the theorem but not the weak form at $x=0$. We also remark that a function $f$ is differentiable at an interior point $x_{0}$ of an interval $I$ if and only if $\left(f\left(x_{0}+h\right)-f\left(x_{0}-k\right)\right) /(h+k)$ has a limit, i.e., $f^{\prime}\left(x_{0}\right)$, when $(h, k)$ $\rightarrow(0,0)$ in the set of pairs such that $h>0, k>0$. But it need not be true that $\left(f\left(x_{0}+h\right)-f\left(x_{0}+k\right)\right) /(h-k)$ has a limit when $(h, k) \rightarrow(0,0)$ in the set of pairs such that $h>0, k>0, h \neq k$. The function $f: R \mapsto R$ defined by $f(x):=$ $x^{2} \sin (1 / x)$ for $x \neq 0$ and $f(0):=0$ illustrates this [2, p. 150].

\section{REFERENCES}

1. A. M. Bruckner, J. B. Bruckner, B. S. Thomson, Real Analysis, Prentice-Hall, Inc. 1997.

2. J. Dieudonné, Foundations of Modern Analysis, Academic, New York, 1960.

3. K. R. Stromberg, An Introduction to Classical Real Analysis, Wadsworth International Mathematics Series, 1981.

4. J. Tong, P. A. Braza, A converse of the mean value theorem, Amer. Math. Monthly 104 (1997), 939-942.

Center for Experimental and Constructive Mathematics, Department of Mathematics and Statistics, Simon Fraser University, Burnaby, BC. V5A IS6, Canada jborwein@cecm.sfu.ca, xwang@cecm.sfu.ca 